\title{
The role of the pharmacist in emergency contraception
}

High street pharmacies have always played a key role in helping people to look after their own health, including sexual health. Women routinely visit pharmacies to purchase or seek advice from pharmacists on treatments for minor ailments such as vaginal thrush, some forms of contraception, and for the dispensing of prescriptions for contraceptive products. Many men use them routinely to access condoms. The accessibility of pharmacies is an acknowledged strength; so it was deeply frustrating for all concerned that prior to 2001, women asking for emergency hormonal contraception (EHC) needed to be referred to other services (often incurring a significant delay). Happily the reclassification of Levonelle (levonorgestrel) as a pharmacy medicine in January 2001 has allowed women to buy this product over the counter from pharmacies as Levonelle (£24/\$37£ 39), and professional guidance reflecting Family Planning and Reproductive Health (FPRH) Guidelines is available at www.rpsgb.org.uk/pdfs/ehcguid.pdf.

The first services utilising patient group directions (PGDs) for EHC in Health Action Zones (HAZs) in Manchester and South London have been in operation for over 2 years, and evaluation data is available at www.rpsgb.org.uk/nhsplan/pgd.htm. ${ }^{1}$ The evaluation of the HAZ service in Lambeth, Southwark and Lewisham showed that $98 \%$ of users were either satisfied or very satisfied with the service, and $90 \%$ had felt comfortable or very comfortable discussing their request with the pharmacist. Although $77 \%$ of users felt that there was enough privacy to talk to the pharmacist comfortably, there is still scope for improvement. In Lambeth, Southwark and Lewisham, a consultant within Reproductive Health Services has led the pharmacists' training programme, and is available via a mobile phone for query answering (see article by Bacon et al. on pp. 17-22). This approach has ensured that the service and information that women receive from pharmacists is consistent with other providers. Some perceived barriers have needed to be addressed, namely dedicated training for the accredited pharmacists, application of Fraser guidelines to consultations with women aged under 16 years, and meeting criteria for private consultation areas. These early services have been extremely successful and are now widely replicated.

One criticism heard is that only accredited pharmacies and pharmacists are able to provide EHC free under the National Health Service (NHS), which is seen as inequitable. Although true, services are planned to provide maximum access in areas of greatest need. The Utopian view that all pharmacies should offer this service is unlikely to be achievable, since not all pharmacies have permanent staff, and very substantial increases in NHS funding would be needed.

There are clearly now more choices available for women to access EHC promptly, both through pharmacies and from other providers. Commissioners can usefully consider the underlying reasons affecting women's choice of provider, which may include location, waiting times, convenient opening hours, feeling comfortable with staff, confidentiality, wish for anonymity, cost and lack of awareness of all available services.

A recent audit by the Lambeth, Southwark and Lewisham Clinical Governance Resource Group ${ }^{2}$ has compared EHC services provided by general practitioner (GP) practices, family planning clinics (FPCs) and services, community pharmacies working with PGDs, and from minor injuries units/accident and emergency departments against common standards. Results demonstrate that women see pharmacies as offering advantages of location, convenience and opening times, and meeting the service standards set. Pharmacies showed the highest level of adherence in the audit to the standard set for record keeping. The HAZ evaluation showed that over $70 \%$ of women were accessing the service within 24 hours of unprotected intercourse when treatment is known to be more effective. This was significantly higher than for those accessing GPs and family planning services.

One of the successes of the PGD pilots has been to demonstrate that the contraceptive services that pharmacists provide can be developed alongside NHS services and to similar standards. Two-way referrals are now routine. GP practices, NHS Direct and accident and emergency departments often refer patients to pharmacies which are open extended hours including at weekends and bank holidays, while pharmacists are referring women to GPs and FPCs for ongoing contraception, and for clinical reasons arising with the PGD. At a local level, new networks are being forged as a result of this development: a pharmacist might be contacted by a headmistress seeking advice on access for a girl who is under 16, and pharmacists have needed to swiftly refer women to specialist services following reported sexual abuse. Pharmacists have greatly valued direct access to a consultant via a mobile phone; this is a 'first' for them, and confirms the link with a wider team. In all cases, the EHC service has been a catalyst for pharmacy's greater engagement with local NHS services.

What of the future? If these new links with other sexual health providers are working so well, how can they be exploited further? For the small number of women who may make inappropriate use of EHC services it is a logical step to provide access to progestogen-only pills and possibly also combined oral contraceptives through pharmacies, using PGDs that parallel those in other services. Other creative new partnerships with pharmacists could emerge; the easy access and relative anonymity of pharmacies could be attractive locations for some sexually transmitted infection services.

The National Sexual Health Strategy ${ }^{3}$ promotes pharmacy-based EHC services. The next logical step is use similar Level 1 and 2 definitions to develop a new range of sexual health services in pharmacies, in partnership with the NHS.

Statements on funding and competing interests

Funding. Funding was received from Health Action Zone.

Competing Interests. Schering Health Care Ltd provided an educational grant towards pharmacists' training and printing costs.

Beth Taylor, BSc, MRPSGB

Manager, Community Services Pharmacy Team, Southwark PCT, St Giles, St Giles Road, London SE5 7RN, UK. Email:beth.taylor@southwarkpct.nhs.uk

References

1 An evaluation of service users' views on accessing emergency hormonal contraception through accredited community pharmacies using a patient group direction. Lambeth, Southwark and Lewisham Health Action Zone, June 2001.

2 Emergency hormonal contraception access and provision interface audit (Part 2). Clinical Governance Review Group, South East London Shared Service Partnership, 2003.

3 Department of Health. National Strategy for Sexual Health and HIV. London: Department of Health, July 2001. 